\title{
Fractal dimension analysis for seismicity spatial and temporal distribution in the circum-Pacific seismic belt
}

\author{
Lirong Yin ${ }^{1,2}$, Xiaolu Li ${ }^{1,3, *}$, Wenfeng Zheng ${ }^{4}$, Zhengtong Yin ${ }^{5}$, Lihong Song ${ }^{4}$, \\ LiJun Ge $\mathrm{G}^{4}$ and QingChuan Zeng ${ }^{4}$ \\ ${ }^{1}$ Chongqing Engineering Research Center for Remote Sensing Big Data Application, School of Geographical \\ Sciences, Southwest University, Chongqing 400 715, P. R. China. \\ ${ }^{2}$ Department of Geographical and Sustainability Sciences, University of Iowa, Iowa City, IA 52242, USA. \\ ${ }^{3}$ Research Base of Karst Eco-environments at Nanchuan in Chongqing, Ministry of Nature Resources, \\ School of Geographical Sciences, Southwest University, Chongqing 400 715, P. R. China. \\ ${ }^{4}$ School of Automation, University of Electronic Science and Technology of China, Chengdu 610 054, P. R. China. \\ ${ }^{5}$ School of Resources and Environment, Guizhou University, Guiyang 550 025, P. R. China. \\ ${ }^{*}$ Corresponding author. e-mail: xiaoluli.ruth@gmail.com
}

MS received 25 October 2016; revised 16 July 2017; accepted 17 April 2018; published online 4 January 2019

In this study, we present the fractal characteristics of the spatio-temporal sequence for seismic activity in the circum-Pacific seismic belt and vicinity regions, which is one of the most active seismic zones worldwide. We select the seismic dataset with magnitude $M \geq 4.4$ in the circum-Pacific seismic belt region and its vicinity from $1900-2015$ as the objects. Based on the methods of capacity dimension and information dimension, using $\ln (1 / \delta)-\ln N(\delta)$ of the relationship to evaluate and explain, the results show that (1) in the circum-Pacific seismic belt and the surrounding areas, for the seismic activity with magnitude $M \geq 4.4$, the time series dimension is 0.63 , the spatial distribution dimension is 0.52 and they have fractal structure. (2) For the earthquakes with $M \geq 7.0$, the time series dimension increases greatly, which indicates that the cluster characteristics in time is greatly reduced. And the earthquakes with magnitude $7.0 \geq M \geq 4.4$ have significant impact on the characterized by clustering in time in the study region. (3) There is significant fractal structure at spatio-temporal distribution of earthquakes in the circum-Pacific seismic belt. It reveals the tectonic movements keep continuous, obvious anisotropism characteristic of geological structure and the distribution of surface stress field is spatio-temporal heterogeneity in the study area.

Keywords. Fractal dimension analysis; circum-Pacific seismic belt; spatio-temporal distribution.

\section{Introduction}

As an important branch of non-linear science, fractal theory can be used to reveal the characteristics of non-stability and discontinuity in the complex system (Higuchi 1988; Goldberger 1996). At present, the fractal theory has been applied to the fields of chemistry, geochemistry, biophysics, geology, geophysics (Birdi 1993; Turcotte 1997; Dimri 2000) and the application in geography is still in exploring stage. The research can further promote the application of fractal theory in the study of geography (Padhy et al. 2015). As its greatest advantage in studying the spatialtemporal propagation characteristics of seismic activities, fractal theory is not limited on the 
cognition of the earth's interior (Ogata and Abe 1991). We can study on earth plate movement and seismic activity in a new perspective by the fractal theory.

The circum-Pacific seismic belt is the high incidence area of earthquakes and about $80 \%$ of the earthquakes in the world occur in this area. The high frequency and high intensity seismic activity has caused serious damage at the area and vicinity regions (Zheng et al. 2017). In recent years, many researchers have paid closed attention to the area.

Most of the researches on seismicity spatiotemporal sequences (Zhuang et al. 2002) are based on the former seismic models to analyze or predict, and are deficient in seismic theory and complexity of geographical structure. It is difficult to obtain the spatio-temporal characteristic of seismic activity completely. From the aspects of magnitude and intensity, fractal theory presents a new avenue for studying the spatio-temporal distribution of earthquakes. In the 1980's, Kagan and Kuopoff (Kagan 1991) applied the fractal theory to the study of the spatial distribution of the epicenter. The results show that there are scale invariance and fractal structure with the spatio-temporal sequence. In the early 1990's, the Soviet Union Geilikman (Geilikman et al. 1990), selected seismic data set of the Pamir-Tianshan, Caucasus and California regions, and found that there are variability among areas in the discontinuity, sparsely and densely sectors characteristic of singular spectrum and fractal dimensions of seismic activities, which could be used as indicators for prediction. In sum, fractal theory can be used to study the spatial-temporal distribution characteristics of earthquakes.

As its research object, this paper selects the original seismic dataset from 1900 to 2015 in the circum-Pacific seismic belt. From the aspects of time series and space distribution sequences, the spatial-temporal distribution of seismic activities in the circum-Pacific seismic belt are analysed by fractal theory to provide a theoretical basis for the prevention and exploration of earthquakes.

\section{Fractal dimension}

Fractal dimension is an important index to quantitatively describe the fractal. It is a measurement in self-similar characteristic with the complexity degree of system. It is a key factor to distinguish different shape complexity ( $\mathrm{Li}$ and $\mathrm{Wu}$
2002). Although the fractal theory is still in the starting stage about the study of geological disasters, it is an inevitable trend to combine the two. Fractal theory will be an important starting point for the analysis of spatio-temporal distribution characteristics in seismic activity (Dimri 2005). In the study of seismic activity, the capacity dimension and the information dimension in the fractal dimension can reflect the fractal characteristics of the spatio-temporal distribution sequence by the spatial correlation scale and the pattern intensity (Sunmonu et al. 2001). The fractal feature of seismic activity is the random self-similar structure of the earthquake in the time series and the spatial distribution sequence, and the larger capacity dimension value indicates that the seismic activity is more active in the time series, and the seismic activities are more concentrated in the spatial distribution and vice versa ( $\mathrm{Li}$ and $\mathrm{Wu} 2002$ ). Based on fractal theory, in this study, therefore, it aims to analyze the spatio-temporal distribution characteristics of the seismic activity in the seismic belt from 1900 to 2015 by the method of capacity dimensions and information dimensions.

Using the box counting dimension, the study researches fractal characteristic of the seismic spatio-temporal space in the circum-Pacific seismic belt by calculating capacity dimension and information dimension of the time series and the spatial distribution in the seismic activity. Each seismic activities are set as a data point in the seismic dataset; $\delta$ as minimum spatio-temporal interval. With the minimum spatio-temporal scale $\delta, N(\delta)$ is number of non-empty subsets under the whole seismic dataset. As the $\delta$ value head towards zero, the fractal dimension is calculated. The capacity dimension $D_{c}$ set as (Sunmonu et al. 2001):

$$
D_{c}=-\lim _{\delta \rightarrow 0} \frac{\ln N(\delta)}{\ln (\delta)} .
$$

The capacity dimension values present the degree of activity of the earthquake in time.

When the $i^{\text {th }}$ component contains the number of data points as $N_{i}(\delta)$, the probability for a point data belongs to the $i^{\text {th }}$ subset with the spatiotemporal scale $\delta$, is defined as (Sunmonu et al. 2001):

$$
p_{i}(\delta)=\frac{N_{i}(\delta)}{N(\delta)} .
$$

The information dimension $D_{i}$ is (Sunmonu et al. 2001): 


$$
D_{i}=-\lim _{\delta \rightarrow 0} \frac{1}{\ln \delta} \sum_{i}^{N} P_{i}(\delta) \ln \frac{1}{P_{i}(\delta)} .
$$

The information dimension is closely related to the density level of the earthquake in the spatial sequence.

\section{Study area}

As one of the largest and most seismically active regions in the world, around $80 \%$ earthquake activities in the world occur in the circum-Pacific seismic belt region and in its vicinity (Duda 1965). Most of the seismic activities in the area are shallow and high-frequency. Thereby, the seismic activities is a serious threat for the area. The research selects the circum-Pacific seismic belt as the study area. It is analysed in this study that the spatiotemporal distribution of the seismic activities in this area for further understanding the occurrence mechanism and attenuation law of seismic activities. There is notable significance in studying the seismic activities in the study area.

All original seismic data used in this research were collected from the earthquakes hypocentre file of the United States Geological Survey (USGS). These dataset covered $70^{\circ} \mathrm{N}-50^{\circ} \mathrm{S}$ latitude and $100^{\circ} \mathrm{E}-50^{\circ} \mathrm{W}$ longitude from 1900 to 2015 (figure 1).
For all the original seismic data from 1900 to 2015 in the circum-Pacific seismic belt and its vicinity region, according to the GutenbergRichter's law (Zheng et al. 2017), the minimum magnitude is 4.4 after the integrity analysis. And the number of magnitude $M \geq 4.4$ seismic activities in this area is 65,535 , which is far beyond the minimum number requirements of data points (50 data points) (Wang and Lee 1997). In other words, our study data, the magnitude $M \geq 4.4$ earthquakes in the study area collected from USGS database, are sufficient for the fractal dimension analysis. Therefore, in this study we select earthquakes from 1900 to 2015 , with $M \geq 4.4$ level as the sample for subsequent analysis.

\section{Fractal analysis of seismic temporal sequence}

As there is continuous movement and collision of plates, the longer the intervals between two adjacent earthquakes, the more energy builds up and unleash. Therefore, the time interval between two adjacent seismic activities reflects the temporal characteristics of the earthquakes in a certain extent. The seismic activity in the study area can be regarded as a discontinuous point set with seismic attributes. Each time point corresponds to an earthquake. Accordingly, the time interval between

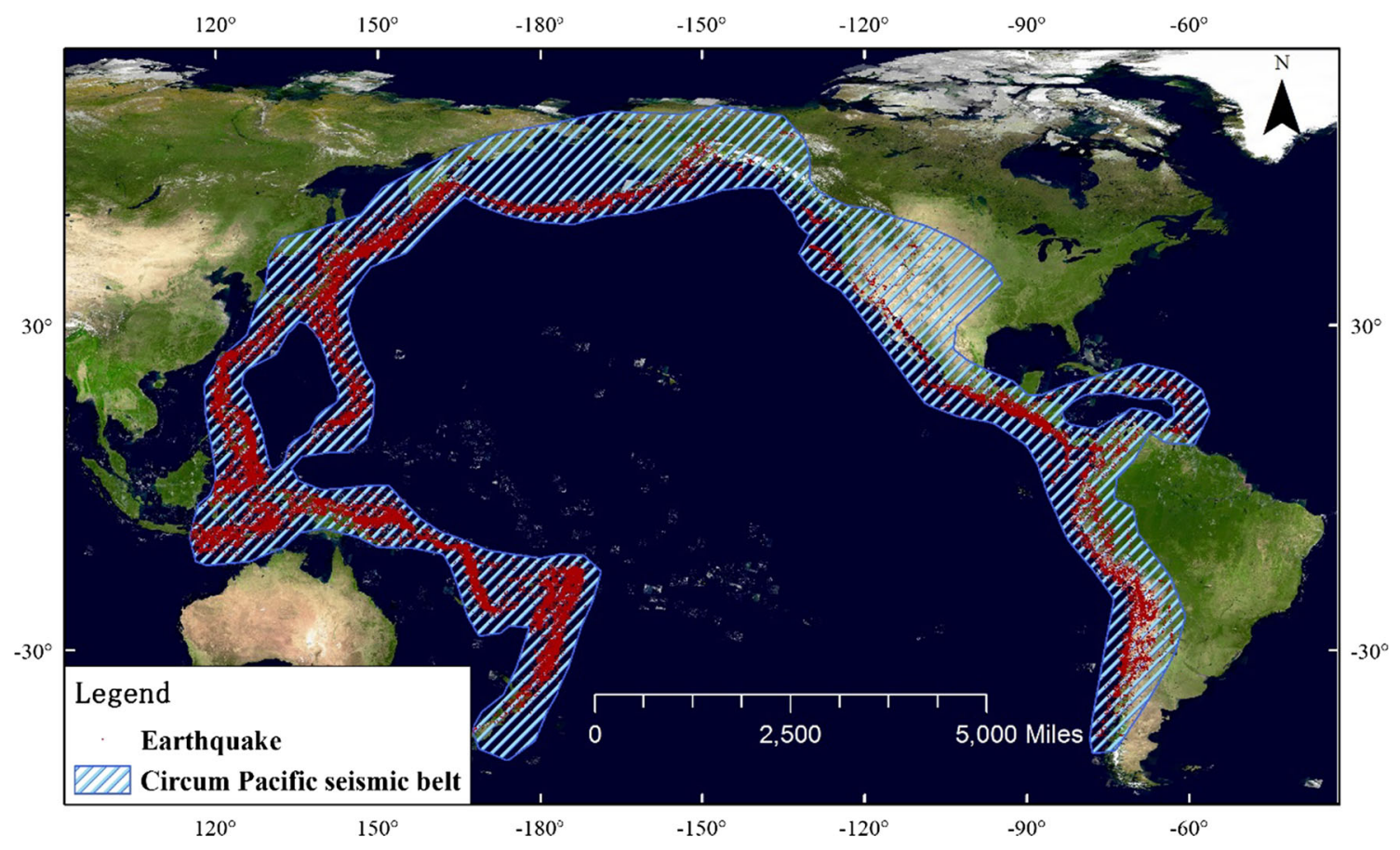

Figure 1. Earthquake distribution in the study area between 1900 and 2015. 


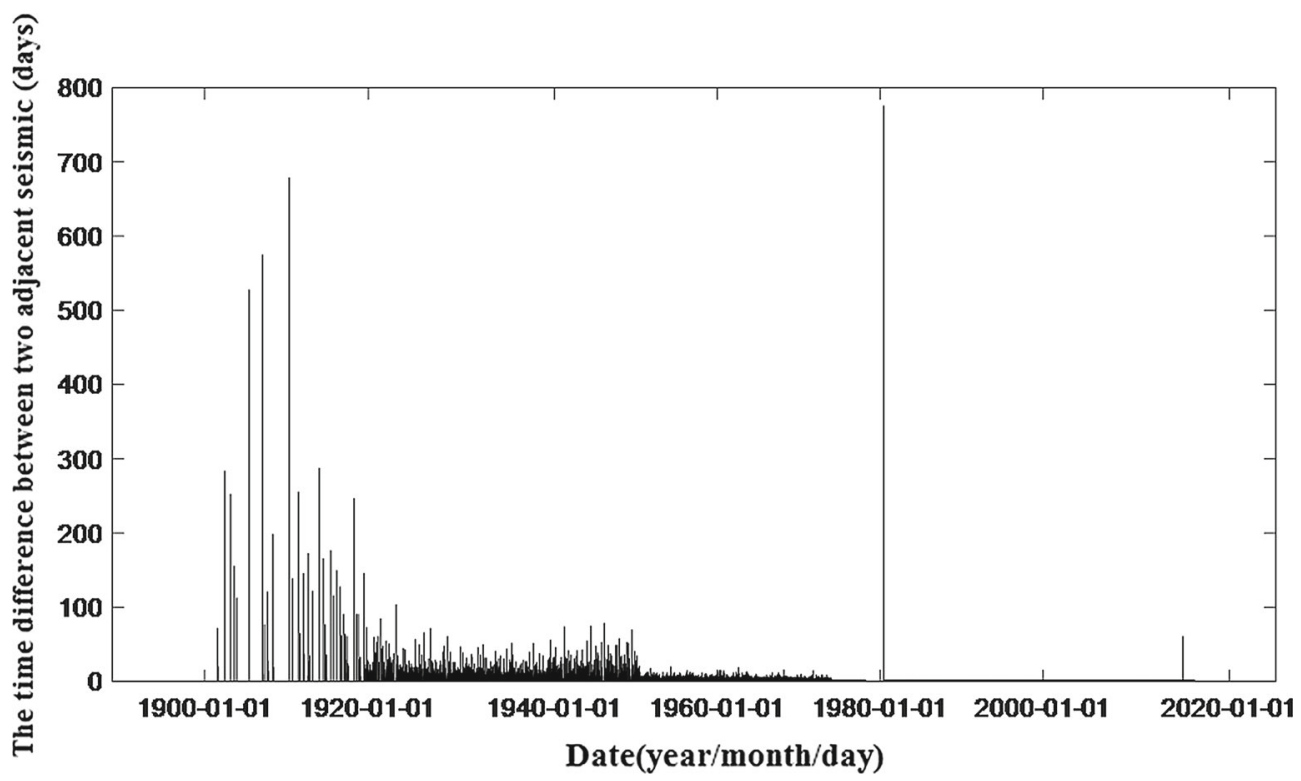

Figure 2. Time interval map of adjacent earthquakes in the study area.

the seismic activities in the research area was counted (figure 2). After the seismic data of the study area are pre-treated, the changes of the time interval are fluctuation over time. The trend of time interval is drastically reduced, and finally tends to be stable. As shown in figure 2, the maximum time interval for adjacent earthquakes is around 1980, while the longer interval is more concentrated from 1900 to 1920. Between 1900 and 1975, the frequency of earthquakes was decreased, and after 1975, the frequency of seismic activity increased gradually. Seismic activity is heterogeneous in time dimension.

In this study, each seismic activity is regarded as a data point in the study area, and $\delta$ represents the minimum seismicity time interval that is not zero, called the minimum time scale. At the minimum time scale $\delta, N(\delta)$ is the minimum number of covering required to cover all seismic activity time series. The capacity dimension $D_{c}$ of the seismic activity time series of the study area is calculated by the formula (1), shown as $\ln (1 / \delta)-\ln N(\delta)$ in figure 3.

The $\ln (1 / \delta)-\ln N(\delta)$ curve of the seismic activities has a turning point, which acts as the demarcation point of the non-scale region. It indicates the presence of fractal structures in the seismic time series of the study area. The capacity dimension $D_{c}$ is a quantitative parameter that measures the fractal structure of fractal objects. The slope of the non-scale region has a capacity dimension of 0.63 , thereby, indicating the seismicity appears to be active in time.
From the $\ln (1 / \delta)-\ln N(\delta) \quad$ curve, with the increase of $\ln (1 / \delta)$ value, $\ln N(\delta)$ is increscent primarily and latterly in a steady state. It suggests that the seismic frequency increases rapidly, then increase slowly, and then almost without an increase.

Many small earthquakes occur before and after a strong earthquake, thereby indicating a certain relationship between these earthquakes. To analyse such relationships, seismic data with the magnitude $M \geq 7.0$ were extracted and analysed via fractal analysis. The interval time of earthquakes with high-magnitude $(M \geq 7.0)$ shows the uneven distribution of seismic activities in time in this area.

The time points of the large earthquakes in the study area were used as interval points to partition the seismic activities in the entire seismic zone. The information dimension (equation 2) of a small earthquake swarm in different segments were calculated and analysed to determine the relationship between strong and small earthquake swarms (figure 4). The fractal dimension value of seismic activities greatly fluctuated along with time, especially around 1930, 1940, and 1990. This indicates that the seismic activity in the study area is more regular in these time periods. It could be noticed that prior to each peak value, the fractal dimension reaches the minimum. The change dramatically from the early minimum to later peak value, it reveals the seismic alternatively phenomenon of the active and quiet period. And also shown in figure 4 , the fractal dimension attenuate sharply 


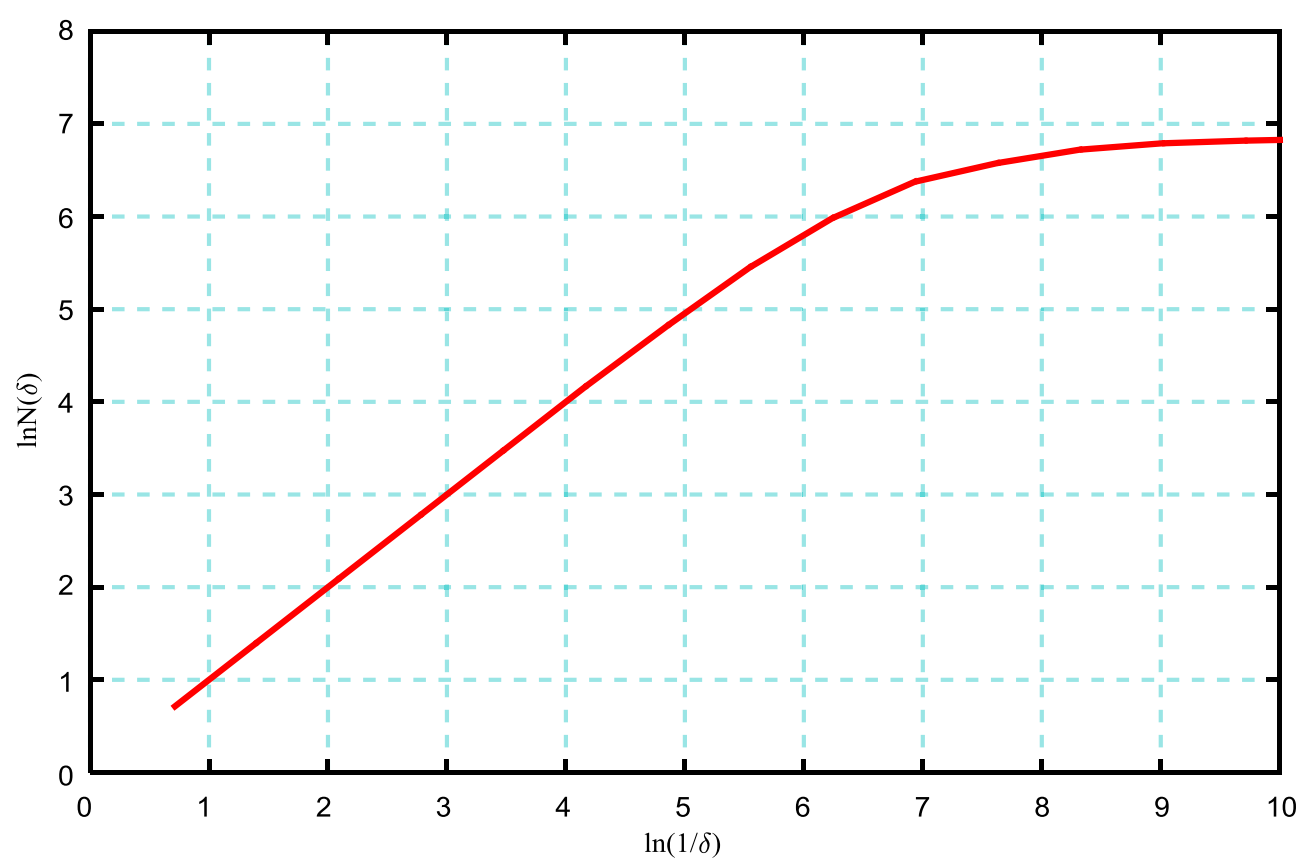

Figure 3. $\ln (1 / \delta)-\ln N(\delta)$ curve of the seismic temporal distribution for the capacity dimension.

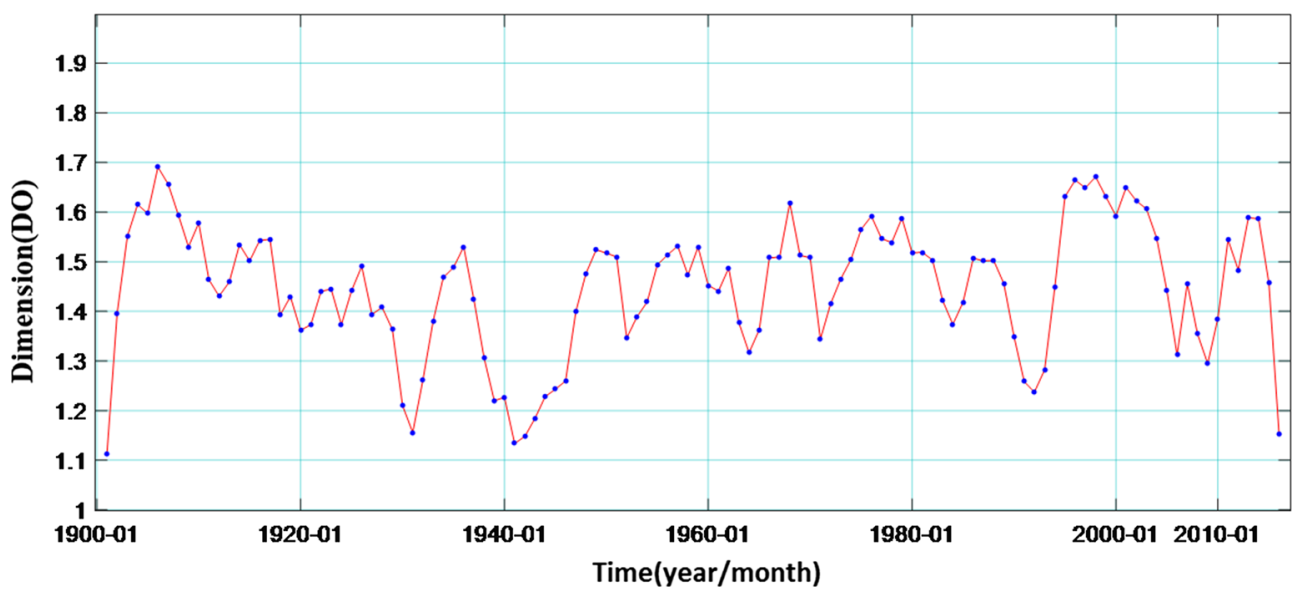

Figure 4. Plot of the information fractal dimension of the independent earthquake swarm in the study area.

after reaching its peak. It could be explained by the release of energy after seismic activities in active periods.

\section{Fractal analysis of the spatial distribution sequence in the study area}

In the fractal analysis of the seismic spatial distribution in the study area, our research use grid transformation to process the seismic dataset of which magnitude is greater than 4.4 and spatial distribution sequence in the area is from 1900 to 2015 as the dataset pre-processing. By means of grid transformation, all seismic point data in the study area are divided into different grid areas according to the spatial distribution. That means all the data are divided into sub-datasets. Therefore, in the study of fractal analysis of spatial distribution, the minimum grid scale that seismic activity area is not empty is chosen to be the value of $\delta$, that is the smallest grid scale. The value of the smallest grid scale that we choose in this research is $0.4^{\circ} . N(\delta)$ means the minimum coverage number, which is required to cover the spatial distribution of all seismic activity at the minimum grid scale $\delta$. The capacity dimension of the spatial distribution of seismic activity in the study area which is called $D_{c}$ is calculated by equation (1) shown in figure 5 .

Compared with the result of fractal dimension analysis, the plot of $\ln (1 / \delta)-\ln N(\delta)$ between the 


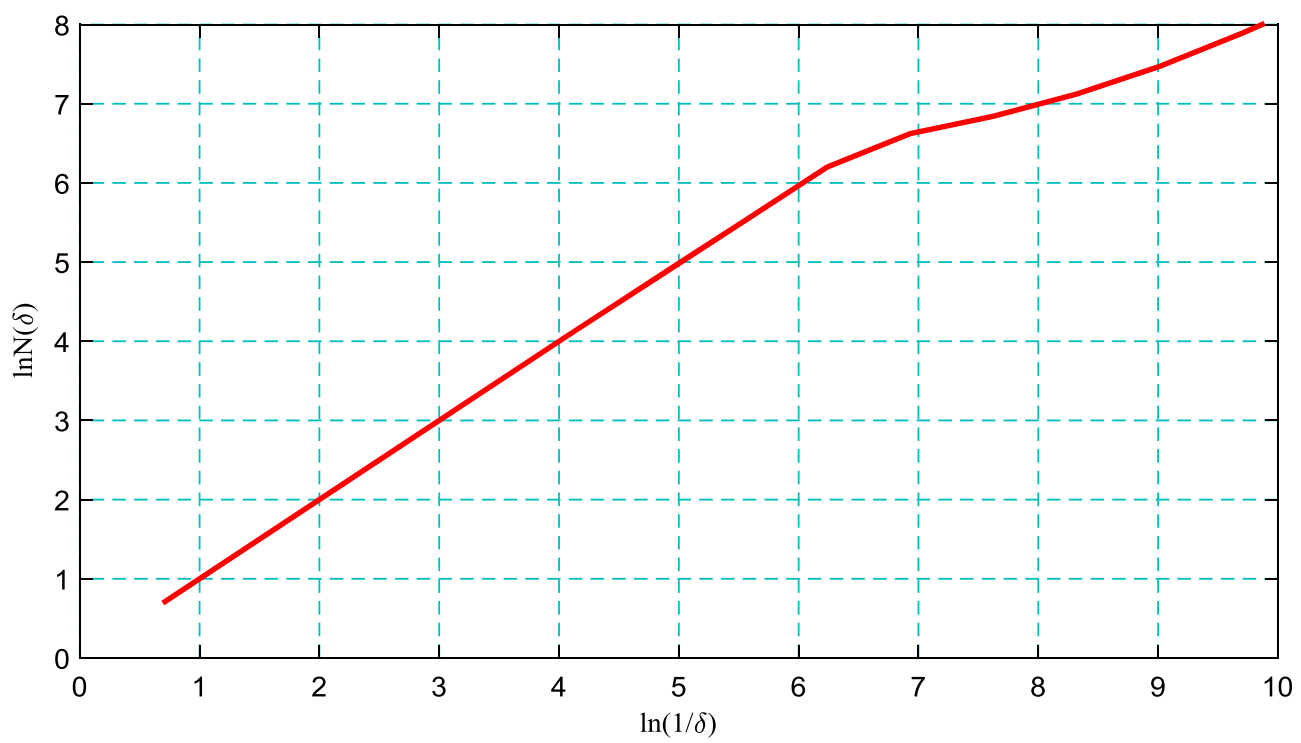

Figure 5. $\ln (1 / \delta)-\ln N(\delta)$ curve of the seismic spatial distribution for the capacity dimension.

temporal sequence and spatial distribution for the seismic dataset (figures 3 and 5) shows that

(1) The trends of the two curves are similar and there are inflection points. The seismic activities in the study area is divided into scale region and non-scale region by the points.

(2) There is a second inflection point in the curve of the spatial distribution sequence of $\ln (1 / \delta)-$ ln $N(\delta)$. Non-scale region exists between the two turning points. The double logarithm graph shows a complex change, which indicates that the fractal structure of the seismic activity is highly complex and variable in the spatial distribution sequence.

(3) The slope of the non-scale region of the seismic activity in the spatial distribution sequence is obtained via one element linear regression. The spatial distribution sequence has a capacity dimension of 0.52 , and the time dimension of the time series is 0.63 . It shows that the self-similarity in spatial distribution is more obvious than the time series, and the seismicity shows the characteristics of temporal and spatial agglomeration in the study area.

\section{Discussion and conclusion}

As one of the largest earthquake belt in the world, the circum-Pacific seismic belt, it has a profound influence on the rim of the Pacific Ocean and surrounding areas. In the study, using the fractal theory, it analyses the spatio-temporal distribution characteristics of the seismic activities from 1900 to 2015 , to explore the spatio-temporal variation mechanism and law of seismic activities in the area.

(1) Some self-similarity and non-scale region exit in the time and space series of the seismic activities. In this research, the fractal method is used to analyse the temporal and spatial distribution characteristics of the earthquake in the study area, and there are significant inflection points in the $\ln (1 / \delta)-\ln N(\delta)$ graph, which is the demarcation point of the nonscale region. The earthquakes in the study area are self-similar in the space and time. The slope of the curve of $\ln (1 / \delta)-\ln N(\delta)$ represents the capacity dimension. The capacity dimension on the spatio-temporal distribution sequence is greater than 0.5 , which suggests that the seismic activity in the time series is active and clustered on the spatial distribution sequence.

(2) It shows that there is a close spatial and temporal clustering relationship between the great earthquake and the before-and-after small earthquakes. After fractal analysis of the $M \geq$ 7.0 earthquake in the area, it can be seen that the fractal dimension of the earthquake group is from 1.1 to 1.7. Compared with the fractal dimension of the earthquakes with $M \geq$ 4.4 (the value is 0.63 ), there is a significant increase. The clustering characteristics of the seismic activity in the study area are mostly caused by the cluster characteristics of weak earthquakes $(4.4 \leq M<7.0)$. 
(3) The fractal structure of the seismic activity in the spatial distribution of the study area is more complicated than that in the time series. According to the spatio-temporal distribution of the earthquake activity in figure $\ln (1 / \delta)-$ ln $N(\delta)$, the fractal structure in the time series linearly increases and then reaches a steady state, that means there is a clear turning point. In the spatial distribution, there is also a turning point, but the difference is that after the turning point there is an approximately rising stage. This indicates that the fractal structure and heterogeneity feature in space is more prominent than in time. It also shows that the anisotropy of the geological structure of the study area is more obvious, and the distribution of surface stress field is with a high degree of heterogeneity in the study area.

\section{Acknowledgements}

Research supported by Fundamental Research Funds for the Central Universities (Nos. SWU117063, SWU116183 and XDJK2011C086); Funds for International S\&T Cooperation and Exchange R\&D Project of Sichuan Province (Grant No. 2017HH0054); Opening Fund of State Key Laboratory of Virtual Reality Technology and Systems (Beihang University) (Grant No. BUAA-VR$16 \mathrm{KF}-11)$.

\section{References}

Birdi K S 1993 Fractals in chemistry, geochemistry, and biophysics: An introduction, Springer Science \& Business Media.

Dimri V P (ed.) 2000 Application of fractals in earth sciences; CRC Press.

Corresponding editor: N V CHALAPATHI RAO
Dimri V P 2005 Fractals in geophysics and seismology: An introduction; In: Fractal Behaviour of the Earth System (ed.) Dimri V P, Springer, New York, 207p.

Duda S J 1965 Secular seismic energy release in the circumPacific belt; Tectonophys. 2(5) 409-452.

Geilikman M B, Golubeva T V and Pisarenko V F 1990 Multifractal patterns of seismicity; Earth Planet. Sci. Lett. 99(1) 127-132.

Goldberger A L 1996 Non-linear dynamics for clinicians: Chaos theory, fractals, and complexity at the bedside; Lancet 347(9011) 1312-1314.

Higuchi T 1988 Approach to an irregular time series on the basis of the fractal theory; Physica D 31(2) 277 283.

Kagan Y Y and Jackson D D 1991 Long-term earthquake clustering; Geophys. J. Int. 104(1) 117-134.

Li S and Wu G T 2002 Fractal and wavelet; Science Press, Beijing, pp. 208-226.

Ogata Y and Abe K 1991 Some statistical features of the long-term variation of the global and regional seismic activity; Int. Stats. Rev. 59(2) 139-161.

Padhy S, Mishra O P, Subhadra N, Dimri V P, Singh O P and Chakrabortty G K 2015 Effects of errors and biases on the scaling of earthquake spatial pattern: Application to the 2004 Sumatra-Andaman sequence; Nat. Hazards 77(1) $75-96$.

Sunmonu L A, Dimri V P, Prakash M R and Bansal A R 2001 Multifractal approach to the time series of $\mathrm{M}>7.0$ earthquake in Himalayan region and its vicinity during 1985-1995; J. Geol. Soc. India 58(2) 163-169.

Turcotte D L 1997 Fractals and chaos in geology and geophysics; Cambridge university Press.

U.S. Geological Survey, Search Earthquake Catalog, http:// earthquake.usgs.gov/earthquakes/search/.

Wang J H and Lee C W 1997 Multifractal measures of time series of earthquakes; J. Phys. Earth 45 331-345.

Zheng W, Li X, Xie J, Yin L and Wang Y 2015 Impact of human activities on haze in Beijing based on grey relational analysis; Rendiconti Lincei 26(2) 187-192.

Zheng W, Li X, Yin L, Yin Z, Yang B, Liu S, Song L, Zhou Y and Li Y 2017 Wavelet analysis of the temporalspatial distribution in the Eurasia seismic belt; Int. J. Wavelets, Multiresolution and Information Processing 15(3) 1750018.

Zhuang J, Ogata Y and Verejones D 2002 Stochastic declustering of space-time earthquake occurrences; J. Am. Stat. Assoc. 97 369-380. 